\title{
Parametrization of 2-thiouracil and 4-thiouracil in CHARMM all-atom empirical force field
}

\author{
J. Sarzyńska* and T. Kuliński \\ Institute of Bioorganic Chemistry, Polish Academy of Sciences \\ Noskowskiego 12/14, 61-704 Poznań, Poland \\ *e-mail: asias@ibch.poznan.pl
}

(Rec. 21 January 2005)

\begin{abstract}
A new set of force field parameters complementing the CHARMM27 all atom empirical force field for nucleic acids was developed for 2-thiouracil and 4-thiouracil, two naturally modified RNA bases. The new parameters allow for molecular modeling and molecular dynamics simulations of RNA containing 2-thiouracil and 4-thiouracil.
\end{abstract}

Key words: CHARMM, force field, nucleic acids, 2-thiouracil, 4-thiouracil, molecular dynamics, quantum mechanical calculations

\section{INTRODUCTION}

Over the last several years, the main goal of molecular biology became the understanding of the functions of biological molecules in terms of structure, interactions and processes at the atomic level. Beside the experimental techniques, computer modeling and simulations, providing information at atomic level and enhancing the interpretation of the biophysical and biochemical experimental data are widely used in the study of biomolecules. Despite the extremely fast progress of computing power, such calculations have to be based on a compromise between the complexity of the description of the molecular system, the number of atoms included, and the computational time required for a reliable description of the investigated process.

The most efficient and commonly used in studies of large and complex biological systems are the techniques based on empirical force field. The force field comprises the potential energy function $U(\mathbf{R})$ and parameters [1]. The potential energy function is a mathematical equation which allows calculating the potential energy as a function of three-dimensional structure. Parameters are related to the chemical structure of studied molecule. The force field describes entire classes of the molecules with compromised accuracy, as an extrapolation from the empirical data of a representative set of molecules.

The majority of biomolecular simulations were performed with CHARMM [2, 3] or AMBER [4] program. The force fields developed within these programs are referred to as CHARMM and AMBER force field, respectively.

The potential energy for the CHARMM force field has a form:

$$
\begin{gathered}
U(R)=\sum_{\text {bonds }} K_{b}\left(b-b_{0}\right)^{2}+\sum_{\mathrm{UB}} K_{\mathrm{UB}}\left(S-S_{0}\right)^{2}+ \\
+\sum_{\text {angle }} K_{\theta}\left(\theta-\theta_{0}\right)^{2}+\sum_{\text {dihedrals }} K_{\chi}(1+\cos (n \chi-\delta))+ \\
+\sum_{\text {impropers }} K_{\varphi}\left(\varphi-\varphi_{0}\right)^{2}+ \\
+\sum_{\text {nonbonded }}\left(\varepsilon_{i j}\left[\left(\frac{R_{\min _{i j}}}{r_{i j}}\right)^{12}-\left(\frac{R_{\min _{i j}}}{r_{i j}}\right)^{6}\right]+\frac{q_{i} q_{j}}{\epsilon_{D} r_{i j}}\right) .
\end{gathered}
$$

The optimized parameters are equilibrium values of bond length, $b_{0}$, Urey-Bradley 1.3 distance, $S_{0}$, valence angle, $\theta_{0}$, improper torsion angle, $\varphi_{0}$, and force constants of bond, $K_{b}$, Urey-Bradley, $K_{\mathrm{UB}}$, valence angle, $K_{\theta}$, dihedral angles, $K_{\gamma}$, and improper torsion angle, $K_{\varphi}$. The parameters $n$ and $\delta$ in the dihedral term are the multiplicity and phase, respectively. These terms are referred to as internal parameters. Nonbonded parameters including partial atomic charges, $q_{i}$, the Lennard-Jones (LJ) well depth, $\varepsilon_{i j}$, and minimum interaction radius, $R_{\min _{i j}}$ are also optimized. The force field parameters are adjusted to simultaneously reproduce small molecule target data obtained from quantum mechanical (QM) calculations and experiments and experimental results for nucleic acid oligomers, e.g., condensed phase structural properties of DNA and RNA.

The most recent CHARMM force field for nucleic acids is CHARMM27 [5, 6]. The AMBER force field for nucleic acid was developed by Cornell [7] and subtly modified by Cheatham [8]. Both force fields lead to the reliable description of the structure, energetics and dynamics of nucleic acids [9-13]. The AMBER force field does not have 
Urey-Bradley term describing 1-3 interactions between the atoms bonded to the common atom. Furthermore, the improper torsion is described as the standard torsion term. The major difference between these force fields is in the way the parameters of the energy function are derived, although both force fields' development relies on $a b$ inito and experimental target data. In the CHARMM, partial atomic charges are manually adjusted to reproduce a set of $a b$ inito calculated interaction energies between selected model compound and water molecule, whereas in the AMBER force field, partial atomic charges are derived using the restrained electrostatic potential (RESP) approach [14].

The aim of this work is to extend the CHARMM27 force field to be able to treat two chemically modified nucleic acid bases, 2-thiouracil (2SU) and 4-thiouracil (4SU).

The 2SU and 4SU are sulphur-containing analogues of uracil, a natural component of RNA. The 2SU is known to be a minor component of transfer ribonucleic acid (tRNA) [15]. In particular, tRNA(Lys)(UUU) has 2-thiouridine derivatives at wobble position 34 . This modification is essential for Watson-Crick (AAA) and wobble (AAG) cognate codon recognition by tRNA(Lys)(UUU) at the ribosomal aminoacyl and peptidyl sites $[16,17]$. Chemically modified bases, including $2 \mathrm{SU}$ and $4 \mathrm{SU}$ were frequently studied for their numerous pharmacological, biochemical and biological capabilities. The 2SU and 4SU were also studied by theoretical chemists [18-21].

\section{METHODS}

Parameterization of $2 \mathrm{SU}$ and $4 \mathrm{SU}$ was performed according to the previously established protocol for the CHARMM force field [5, 22, 23]. Parameterization starts from the topology and the initial parameters assignment for the selected model compound. For a large molecule this is usually its fragment. Parameter optimization is a multistep process involving iterative recalculations with tuned external and internal parameters. Results obtained in CHARMM force field are compared to the target data from experiment or QM calculations and the parameters are manually adjusted to obtain the best agreement. The target data for charge optimization are the base-water minimum interaction energies and distances calculated $a b$ initio, whereas target data for bond and angle equilibrium values are experimental data or the geometry from $a b$ initio optimization.

The gas phase $a b$ initio calculations were carried out with the Gaussian 03 program [24]. The QM minimum interaction energies and distances between different sites of the studied bases and individual water molecules placed in idealized orientations were determined at the HF/6-31G* level by optimizing the interaction distances. The intra- molecular geometries were constrained to the gas phase HF/6-31G* optimized structure for the base and the TIP3P geometry for the water [25]. The interaction energies were calculated as the total energy of the base-water supramolecular complex minus the sum of the individual monomer energies.

Molecular mechanics calculations in the gas phase were performed with the CHARMM program, version 30, with no truncation of nonbonded interactions and dielectric constant $\varepsilon_{D}$ equal 1. Minimum interaction energies and geometries between bases and water molecules were determined in CHARMM by varying the interaction distances, with the intramolecular geometries constrained to the CHARMM gas phase minimized structure for the bases or the TIP3P geometry for water. The energy minimization involved 50 steps of steepest descent (SD) followed by 20 steps of Nepton-Raphson (NR) minimizer. The orientations of the individual water molecules were identical to those used in the QM calculations.

Total interaction energies for base pairs were determined as the difference between the total energy of the minimized dimer and the sum of the minimized monomer energies. Dimer minimization involved building the dimer from internal coordinates followed by 200 steps of Adopted Basis Nepton-Raphson (ABNR) minimization with harmonic constrains $1.0 \mathrm{kcal} / \mathrm{mol} \AA$ on all nonhydrogen atoms, followed by $200 \mathrm{ABNR}$ steps without constrains.

\section{RESULTS AND DISCUSSION}

To obtain parameters for $2 \mathrm{SU}$ and $4 \mathrm{SU}$, which are compatible to CHARMM27 force field, the same procedure as for the development of the original force field was applied. This also included the choice of $\mathrm{HF} / \mathrm{G}-31 \mathrm{G}^{*}$ level of theory for QM calculations of base-water interactions. The emphasis has been placed on the optimization of the partial atomic charges. The proper representation of the electrostatic interactions is crucial for the force field for biological macromolecules.

The structures and atom numbering of $2 \mathrm{SU}$ is shown in Fig. 1.<smiles>Cc1c(C)n(C)c(=S)n(C)c1=O</smiles>

Fig. 1. Molecular structure and atom numbering of $2 \mathrm{SU}$ 
The initial topology information (connectivity, atom types), and as a consequence most internal parameters for 2SU and 4SU were transferred directly from uracil (URA). For sulphur (S), atomic type found on methanethiol in topology and parameter file for small model compounds used in the development of the CHARMM22 protein allhydrogen parameters was taken, which simultaneously assigned its van der Waals (VDW) parameters. Missing parameters, unique to the new bases, were only bond, angle and torsion parameters associates with the sulphur atom. These parameters were assigned by the analogy to existing parameters. Preliminary partial atomic charges were also derived from URA and expected changes for $2 \mathrm{SU}$ and $4 \mathrm{SU}$, based on the Muliken charges of an $\mathrm{HF} / 6-31 \mathrm{G}^{*}$ calculations for URA, 2SU and 4SU.

The initial parameters were subsequently optimized via an iterative approach. The optimization processes started from the determination of partial atomic charges. The partial atomic charges were optimized to reproduce minimum energies and distances for individual water molecules interacting with different sites on $2 \mathrm{SU}$ and $4 \mathrm{SU}$. Different interaction orientations between $2 \mathrm{SU}$ and water molecules are shown in Fig. 2. The same orientations of the water molecules were used for 4SU. Partial atomic charges were manually adjusted to get the best agreement between the energies and the distances obtained from CHARMM and QM calculation for all base-water interactions. QM energies were scaled by 1.16 factor for all interactions prior to the comparison with CHARMM energies [22]. The next

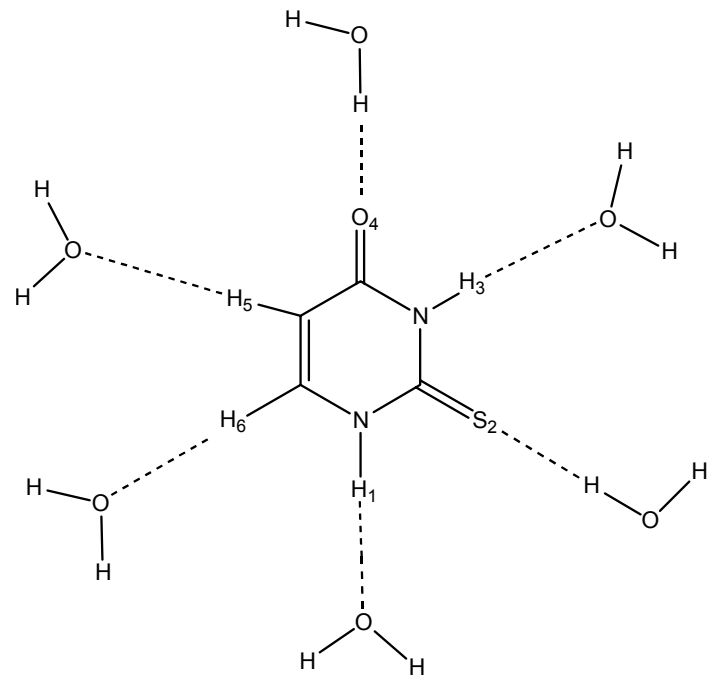

Fig. 2. Interaction orientations between $2 \mathrm{SU}$ and water used in optimization of the partial atomic charges

step in the optimization process involved bond and angle terms. The 2SU and 4SU were energy minimized within CHARMM27 force field using initially assigned bond and angle equilibration values and partial atomic charges obtained in the previous step. The bond and angle equilibration values for newly introduced parameters were manually adjusted to better reproduce target data. The target data for geometry optimization of 2SU and 4SU was the geometry extracted from the crystal structure of

Table 1A. Ab initio and empirical energies and distances between 2SU and water molecule

\begin{tabular}{l|rc|rr|rr}
\hline \multirow{2}{*}{ Interaction } & \multicolumn{2}{|c|}{ Ab initio } & \multicolumn{2}{c|}{ CHARMM27 } & \multicolumn{2}{c}{ Difference } \\
\cline { 2 - 7 } & $E_{\min }$ & $R_{\min }{ }^{*}$ & $E_{\min }$ & -8.692 & 1.79 & \multicolumn{1}{c}{$\Delta E$} \\
\hline H1-OHH & -8.725 & 1.94 & -7.580 & 1.81 & -0.033 & -0.15 \\
H3-OHH & -7.568 & 1.92 & -3.132 & 2.35 & 0.052 & -0.11 \\
H5-OHH & -3.184 & 2.38 & -5.316 & 2.29 & -0.012 & -0.03 \\
H6-OHH & -5.302 & 2.30 & -4.659 & 1.80 & -0.026 & -0.1 \\
O4-HOH & -4.633 & 1.90 & -1.447 & 2.50 & -0.023 & -0.33 \\
S2-HOH & -1.424 & 2.83 & &
\end{tabular}

Energies are in $\mathrm{kcal} / \mathrm{mol}$ and distances are in $\AA . R_{\min }{ }^{*}$ - minimum distances decreased by 0.2 for polar-neutral interactions, $A b$ initio energies are scaled by 1.16. See Fig. 2 for interaction orientations.

Table 1B. Ab initio and empirical energies and distances between 4SU and water molecule

\begin{tabular}{lcccccc}
\hline \multirow{2}{*}{ Interaction } & \multicolumn{3}{c}{ Ab initio } & \multicolumn{2}{c}{ CHARMM27 } & \multicolumn{2}{c}{ Difference } \\
\cline { 2 - 7 } & $E_{\min }$ & $R_{\min }{ }^{*}$ & $E_{\min }$ & $R_{\min }$ & $\Delta E$ & $\Delta R$ \\
\hline H1-OHH & -8.780 & 1.97 & -8.819 & 1.78 & 0.039 & -0.09 \\
H3-OHH & -7.343 & 1.93 & -7.350 & 1.81 & 0.007 & -0.12 \\
H5-OHH & -2.736 & 2.36 & -2.769 & 2.39 & 0.033 & 0.03 \\
H6-OHH & -5.187 & 2.30 & -5.205 & 2.30 & 0.018 & -0.0 \\
O2-HOH & -4.801 & 1.90 & -4.757 & 1.8 & -0.044 & -0.1 \\
S4-HOH & -1.376 & 2.82 & -1.338 & 2.5 & 0.038 & -0.32 \\
\hline
\end{tabular}

Energies are in $\mathrm{kcal} / \mathrm{mol}$ and distances are in $\AA . R_{\min }{ }^{*}$ - minimum distances decreased by 0.2 for polar-neutral interactions, $A b$ initio energies are scaled by 1.16. Orientations of the water molecules were the same as for $2 \mathrm{SU}$ in Fig. 2. 
Table 2. Average differences, RMS differences and average absolute error between the base to water ab initio and empirical interaction energies $[\mathrm{kcal} / \mathrm{mol}]$

\begin{tabular}{c|c|c|c}
\hline Base & $\begin{array}{c}\text { Average } \\
\text { difference }\end{array}$ & RMS difference & $\begin{array}{c}\text { Average absolute } \\
\text { error }\end{array}$ \\
\hline $2 \mathrm{SU}$ & 0.0004 & 0.03 & 0.03 \\
$4 \mathrm{SU}$ & -0.003 & 0.03 & 0.03 \\
\hline
\end{tabular}

Average absolute error is the sum of the absolute values of the differences divided by $n$, the number of interactions of water with each base.

Table 3. Statistical analysis of bond and angle parameters differences with respect to target data

\begin{tabular}{l|cc|cc}
\hline \multirow{2}{*}{ Base } & \multicolumn{2}{|c|}{ Bonds $[\AA]$} & \multicolumn{2}{c}{ Angles [deg] } \\
\cline { 2 - 5 } & $\begin{array}{c}\text { Average } \\
\text { difference }\end{array}$ & SD & $\begin{array}{c}\text { Average } \\
\text { difference }\end{array}$ & SD \\
\hline 2SU & 0.008 & 0.003 & 1.3 & 0.3 \\
4SU & 0.02 & 0.004 & 1.5 & 0.4 \\
\hline
\end{tabular}

SD - standard deviation. Analysis only for bonds and angles involving nonhydrogen atoms. 2-thiouracil [26] and 1-methyl-4-thiouracil [27] respectively. It should be noted that structures minimized in CHARMM do not have bonds lengths and angles that directly correspond to the equilibrium bond and angles parameters.

Following the initial optimization of the bond and angle parameters, partial atomic charges were reoptimized, and then the geometry was rechecked. For $2 \mathrm{SU}$ and $4 \mathrm{SU}$, due to only limited number of new geometry parameters introduced, the convergence in the loop was achieved immediately.

The minimum interaction energies and distances from both the $a b$ initio $\mathrm{HF} / 6-31 \mathrm{G}^{*}$ and CHARMM27 calculations are presented in Table 1A and 1B. Small values of the average differences between base-water QM and CHARMM27 energies $(0.0004 \mathrm{kcal} / \mathrm{mol}$ and $-0.003 \mathrm{kcal} / \mathrm{mol}$ for $2 \mathrm{SU}$ and $4 \mathrm{SU}$ respectively) ensure that the overall solvation of the bases will be reasonable, and small rms differences $(0.03 \mathrm{kcal} / \mathrm{mol}$ and $0.03 \mathrm{kcal} / \mathrm{mol}$ for $2 \mathrm{SU}$ and $4 \mathrm{SU}$ respectively) ensure that no individual term is too far from target data (Table 2).
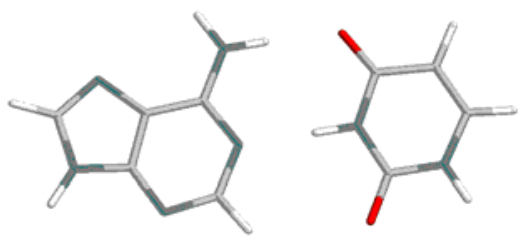

A-U WC<smiles>C=C(C)C1CC2=C(C1C)C(C)C(C)C2C</smiles><smiles>C=C1C(C)=C(C)C(C)C(C)C1C</smiles>

G-U wooble<smiles>C=C(C)C1CC2=C(CC1C)C(C)C(C)C2C</smiles><smiles>C=C1C(C)=C(C)C(C)C(C)C1C</smiles>

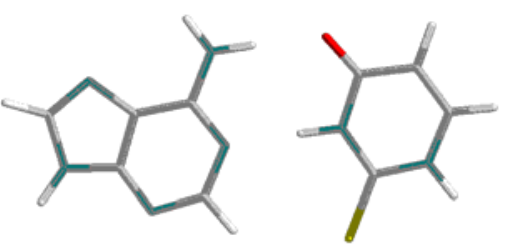

A-2SU WC<smiles>CC(C)C1CC2=C(C(C)C(C)C2C)C(C)C1C</smiles><smiles>C=C1C(C)C(C)C(C)C(C)C1C</smiles>

G-2SU wobble<smiles>CC(C)C1C(C)C2C(C)C(C)C3CC1C(C)C32</smiles><smiles>C=C1C(C)=C(C)C(=C)C(C)C1C</smiles>

Fig. 3. Base pairs structures

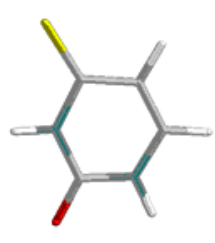

A-4SU WC<smiles>CC1C(C)C(C)C(C)C(C)C1C</smiles>

G-4SU wobble<smiles>CC(C)C1CC2CC(C1)C1CC(C)C(C)CC21</smiles><smiles>CCC1C(C)C(C(C)C)CC2C(C)C(C)CC21</smiles><smiles>CC(C)C1CC2C(C)C(C)CC2C(C)C1C</smiles><smiles>C=C1C(C)C(C)C(C)C(I)C1C</smiles> 
An agreement between CHARMM27 results and target data for the bond and angle equilibrium values was satisfactory when the pyrimidine ring of $2 \mathrm{SU}$ and $4 \mathrm{SU}$ was the same as of URA (Table 3). Better agreement with experimental target data would require the introduction of the new atom types that is not necessary in our case. The bond and angle force constants, all dihedral and improper parameters, were transferred from the existing parameters for uracil.

In addition, the dipole moments are used as a reference data for the optimization of partial atomic charges. In general the dipole moments calculated with CHARMM27 force field are systematically larger than the gas phase QM values due to the lack of induced polarizability in the CHARMM27 force field. Exception is uracil for which dipole moment in CHARMM27 is smaller than QM value [5]. The 2SU and 4SU, similar as URA, have the dipole moments in CHARMM27 smaller than in QM calculations (Table 4)
Table 4. Dipole moments of the 2SU and 4SU from CHARMM27 and $a b$ initio gas phase calculations [Debye]

\begin{tabular}{lccc}
\hline Base & CHARMM27 & HF/6-31G* & MP2/6-31G* \\
\hline URA & 4.29 & 4.72 & 5.03 \\
2SU & 4.33 & 5.32 & 5.35 \\
4SU & 4.05 & 5.67 & 5.67 \\
\hline
\end{tabular}

Optimization of the base parameters also includes base pair interactions. Table 5 reports comparison of the CHARMM27 and the ab initio interaction energies for base dimers presented in Fig. 3. The larger difference occurs for G-2SU and G-4SU wobble base pairs (3.23 and $-2.1 \mathrm{kcal} / \mathrm{mol} \mathrm{respectively).} \mathrm{The} \mathrm{CHARMM} 27$ interaction energies for the 26 base pair defined by Hobza et al. [28] were reported to have a difference up to $2.45 \mathrm{kcal} / \mathrm{mol}$ [5]. The Lennard-Jones (LJ) terms contribute more to the total CHARMM27 interaction energies, when sulphur atom is
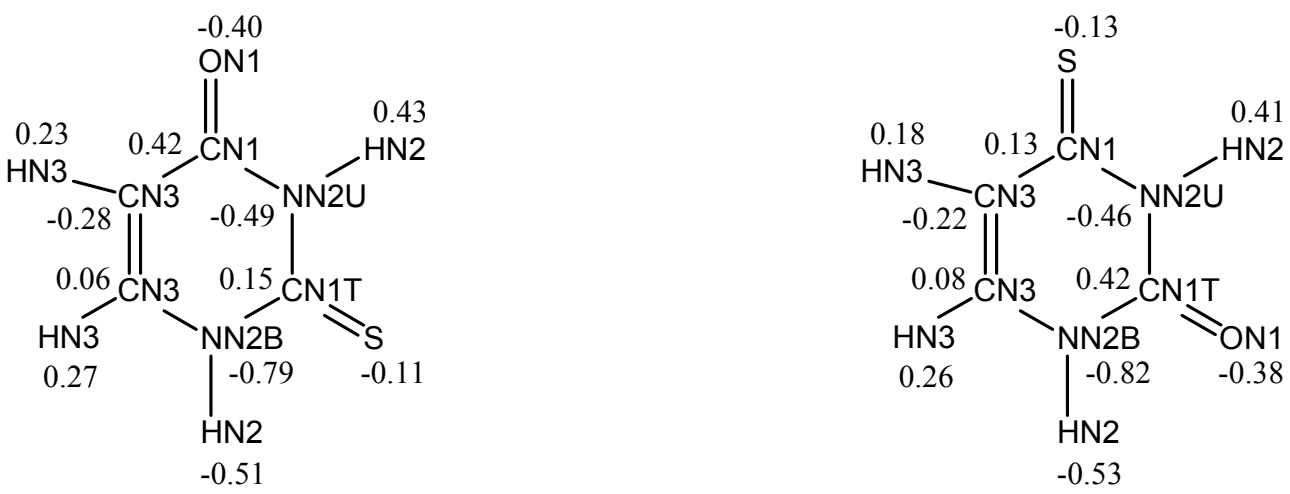

Fig. 4. CHARMM partial atomic charge and atom type parameters for $2 \mathrm{SU}$ and $4 \mathrm{SU}$

Table 5. Comparison of CHARMM27 and ab initio interaction energies for hydrogen bonded base pairs ${ }^{\text {a }}$ [kcal $\left./ \mathrm{mol}\right]$

\begin{tabular}{|c|c|c|c|c|c|c|}
\hline \multirow{2}{*}{ Base pair } & \multicolumn{4}{|c|}{ CHARMM $27^{\mathrm{b}}$} & \multirow[t]{2}{*}{$\mathrm{MP} 2 / / \mathrm{HF}^{\mathrm{c}}$} & \multirow[t]{2}{*}{ Diff. } \\
\hline & Total & Elec & $\mathrm{LJ}$ & Internal & & \\
\hline AU WC & -12.80 & -12.42 & 0.23 & -0.62 & & \\
\hline A2SU WC & -13.24 & -11.96 & -0.52 & -0.76 & $-12.1^{\mathrm{d}}$ & -1.14 \\
\hline A4SU WC & -10.75 & -9.24 & -1.25 & -0.26 & $-11.2^{\mathrm{d}}$ & 0.45 \\
\hline GU wobble & -13.10 & -12.56 & 0.07 & -0.62 & $-12.7^{\mathrm{e}}$ & -0.40 \\
\hline G2SU wobble & -8.87 & -7.78 & -0.97 & -0.11 & $-12.1^{\mathrm{e}}$ & 3.23 \\
\hline G4SU wobble & -14.20 & -13.37 & -0.09 & -0.74 & $-12.1^{\mathrm{e}}$ & -2.1 \\
\hline UU-1 & -10.45 & -10.39 & 0.17 & -0.23 & $-12.1 b^{e}$ & -0.45 \\
\hline 2SU-2SU 1 & -7.59 & -6.14 & -1.33 & -0.12 & $-8.8^{\mathrm{d}}$ & 1.21 \\
\hline 2SU-2SU 2 & -11.34 & -10.90 & 0.15 & -0.27 & $-9.6^{\mathrm{d}}$ & -1.74 \\
\hline
\end{tabular}

a) base pairs are described in Fig. 3;

b) in CHARMM27 force field total interaction energies (Total), electrostatic (Elec), Lennard-Jones (LJ) and internal energy (Internal) contributions are shown;

c) $\mathrm{MP} 2 / / \mathrm{HF}-\mathrm{MP} 2 / 6-31 \mathrm{G}^{*}(0.25) / / \mathrm{HF} / 6-31 \mathrm{G}^{* *}$ level (interaction energies of H-bonded pairs were evaluated at the MP2 level with the $6-31 \mathrm{G}^{*}(0.25)$ basis set using geometry optimised at level $\left.\mathrm{HF} / 6-31 \mathrm{G}^{* *}\right)$ with correction for bases-set superposition error (BSSE);

d) from Table 2 in [18];

e) from Table 3 in [21]. 
involved in hydrogen bonding (A-4SU WC, G-2SU wobble and 2SU-2SU 1 base pairs). These contributions may indicate that the optimization of VDW parameters for the sulphur atom can be considered.

Table 6. CHARMM27 parameters for $2 \mathrm{SU}$ and $4 \mathrm{SU}$. Force constants $K_{b}, K_{\theta}, K_{\chi}$ and $K_{\varphi}$ are in $\mathrm{kcal} / \mathrm{mol} \cdot \AA^{2}$, equilibrium bond lengths $b_{0}$ are in $\AA$, equilibrium bond angles $\theta_{0}, \delta$ and $\varphi_{0}$ are in degrees

Table 6A. Bonds parameters

\begin{tabular}{lcc}
\hline \multicolumn{1}{r}{ Bond type } & $K_{b}$ & $b_{0}$ \\
\hline 2SU & & \\
\hline CN1T S & 480.0 & 1.68 \\
\hline 4SU & & \\
\hline CNT S & 480.0 & 1.67 \\
\hline
\end{tabular}

Table 6B. Angle parameters

\begin{tabular}{lcc}
\hline Angle type & $K_{\theta}$ & $\theta_{0}$ \\
\hline 2SU & & \\
\hline NN2B CN1T S & 100.0 & 122.6 \\
NN2U CN1T S & 100.0 & 123.4 \\
\hline 4SU & & \\
\hline NN2U CN1 S & 100.0 & 121.9 \\
CN3 CN1 S & 100.0 & 125.5 \\
\hline
\end{tabular}

Table 6C. Dihedral parameters

\begin{tabular}{lccc}
\hline Angle type & $K \chi$ & $n$ & $\delta$ \\
\hline 2SU & & & \\
\hline S CN1T NN2B HN2 & 0.0 & 2 & 180.0 \\
S CN1T NN2U HN2 & 0.0 & 2 & 180.0 \\
S CN1T NN2B CN3 & 0.9 & 2 & 180.0 \\
S CN1T NN2U CN1 & 0.9 & 2 & 180.0 \\
S CN1T NN2B CN7B & 11.0 & 2 & 180.0 \\
\hline 4SU & & & \\
\hline S CN1 NN2U HN2 & 0.0 & 2 & 180.0 \\
S CN1 CN3 HN3 & 6.0 & 2 & 180.0 \\
S CN1 NN2U CN1T & 0.0 & 2 & 180.0 \\
S CN1 CN3 CN3 & 1.0 & 2 & 180.0 \\
\hline
\end{tabular}

Table 6D. Improper dihedral parameters

\begin{tabular}{lcc}
\hline Angle type & $K \varphi$ & $\varphi_{0}$ \\
\hline 2SU & & \\
\hline S NN2U NN2B CN1T & 90.0 & 0.0 \\
\hline 4SU & & \\
\hline S NN2U CN3 CN1 & 90.0 & 0.0 \\
\hline
\end{tabular}

The final CHARMM27 charge parameters for 2SU and 4SU are shown in Fig. 4 and the final internal parameters are presented in Table 6 . In the topology of 2SU and 4SU the torsion angles are defined by analogy to those in URA, although force constants for some dihedrals in URA (and as a consequence in $2 \mathrm{SU}$ and $4 \mathrm{SU}$ ) are equal to zero. This is useful if one wants to apply the new developed parameters to free energy calculations for URA $\rightarrow 2 \mathrm{SU}$ or URA $\rightarrow$ 4SU mutations using PERT module in CHARMM. The topology and parameter stream file for 2SU and 4SU to direct use in a CHARMM input script is available on request.

\section{Acknowledgments}

We are indebted to Ansuman Lahiri and Alexander D. MacKerell $\mathrm{Jr}$ for their helpful discussion. Access to the Poznań Supercomputing and Networking Centre has been acknowledged.

\section{References}

[1] A. R. Leach, Empirical Force Field model: Molecular Mechanics (In:) Molecular Molelling: Principles and Applications. Prentice Hall, 165-245, 2001.

[2] B. R. Brooks, R. E. Bruccoleri, B. D. Olafson, D. J. States, S. Swaminathan and M. Karplus, CHARMM: A Program for Macromolecular Energy, Minimization, and Dynamics calculations., J. Comp. Chem. 4, 187-217 (1983).

[3] D. A. MacKerell Jr., B. Brooks, C. L. Brooks II, L. Nilsson, B. Roux and M. Karplus. CHARMM: The Energy Function and Its Parameterization with an Overview of the Program. [In:] P. V. R. Schleyer, N. L. Allinger, T. Clark, J. Gasteiger, P. A. Kollman, H. F. Schaefer III and P. R. E. Schreiner, (ed.) Encyclopedia of Computational Chemistry. John Wiley \& Sons, Chichester, UK, 271-277, 1998.

[4] D. A. Case, D. A. Pearlman, J. W. Caldwell, T. E. Cheatham III, J. Wang, W. S. Ross, C. L. Simmerling, T. A. Darden, K. M. Merz, R. V. Stanton, A. L. Cheng, J. J. Vincent, M. Crowley, V. Tsui, H. Gohlke, R. J. Radmer, Y. Duan, J. Pitera, I. Massova, G. L. Seibel, U. C. Singh, P. K. Weiner and P. A. Kollman, AMBER 7, University of California, San Francisco (2002).

[5] N. Foloppe and A. D. MacKerell, All-atom empirical force field for nucleic acids: I. Parameter optimization based on small molecule and condensed phase macromolecular target data, J. Comp. Chem. 21, 86 (2000).

[6] A. D. MacKerell, N. Banavali and N. Foloppe, Development and current status of the CHARMM force field for nucleic acids, Biopolymers 56, 257-265 (2000).

[7] W. D. Cornell, P. Cieplak, C. I. Bayly, I. R. Gould, K. M. Merz, D. M. Ferguson, D. C. Spellmeyer, T. Fox, J. W. Caldwell and P. A. Kollman, A second generation force field for the simulation of proteins, nucleic acids, and organic molecules, J. Am. Chem. Soc. 117, 5179 (1995).

[8] T. E. Cheatham, P. Cieplak, and P. A. Kollman, A modified version of the Cornell et al. force field with improved sugar pucker phases and helical repeat, J. Biomol. Struct. Dyn. 16, 845-862 (1999).

[9] T. E. Cheatham and M. A. Young, Molecular dynamics simulation of nucleic acids: Successes, limitations, and promise, Biopolymers 56, 232-256 (2000).

[10] W. Wang, O. Donini, C. M. Reyes and P. A. Kollman, Biomolecular simulations: Recent developments in force fields, simulations of enzyme catalysis, protein-ligand, protein-protein, and protein-nucleic acid noncovalent interactions, Annu. Rev. Biophys. Biomol. 30, 211-243 (2001).

[11] J. Norberg and L. Nilsson, Molecular dynamics applied to nucleic acids, Acc. Chem. Res. 35, 465-472 (2002).

[12] M. Orozco, A. Perez, A. Noy and F. J. Luque, Theoretical methods for the simulation of nucleic acids, Chem. Soc. Rev. 32, 350-364 (2003). 
[13] T. E. Cheatham, Simulation and modeling of nucleic acid structure, dynamics and interactions, Curr. Opin. Struct. Biol. 14, 360-367 (2004).

[14] J. M. Wang, P. Cieplak and P. A. Kollman, How well does a restrained electrostatic potential (RESP) model perform in calculating conformational energies of organic and biological molecules? J. Comp. Chem. 21, 1049-1074 (2000).

[15] P. F. Agris, The importance of being modified: Roles of modified nucleosides and $M g 2+$ in RNA structure and function, Progress in Nucleic Acid Research and Molecular Biology, 53, 79-129 (1996).

[16] S. S. Ashraf, E. Sochacka, R. Cain, R. Guenther, A. Malkiewicz and P. F. Agris, Single atom modification $(O$ $>$ S) of tRNA confers ribosome binding, RNA 5, 188-194 (1999).

[17] F. V. Murphy, V. Ramakrishnan, A. Malkiewicz and P. F. Agris, The role of modifications in codon discrimination by tRNA(Lys) UUU, Nature Structural \& Molecular Biology 11, 1186-1191 (2004).

[18] J. Sponer, J. Leszczynski and P. Hobza, Thioguanine and thiouracil: Hydrogen-bonding and stacking properties, J. Phys. Chem. A 101, 9489-9495 (1997).

[19] M. A. Palafox, V. K. Rastogi, R. P. Tanwar and L. Mittal, Vibrational frequencies and structure of 2-thiouracil by Hartree-Fock, post-Hartree-Fock and density functional methods, Spectrochimica Acta Part A. Molecular and Biomolecular Spectroscopy 59, 2473-2486 (2003).

[20] L. A. Eriksson, E. S. Kryachko and M. T. Nguyen, Theoretical study of hydrogenation of thiouracils and their base pairs with adenine, International Journal of Quantum Chemistry 99, 841-853 (2004).

[21] J. Sponer, P. Jurecka and P. Hobza, Accurate interaction energies of hydrogen-bonded nucleic acid base pairs, J. Am Chem. Soc. 126, 10142-10151 (2004).

[22] A. D. MacKerell, Atomistic Models and Force Fields. [In:] O. M. Becker, A. D. MacKerell, B. Roux and M. Watanabe (ed.) Computational Biochemistry and Biophysics. Marcel Dekker, Inc., New York, Basel. pp. 7-38, 2001.
[23] A. D. MacKerell, http://www.pharmacy.umaryland.edu/faculty /amackere/param/force_field_dev.htm

[24] M. J. Frisch, G. W. Trucks, H. B. Schlegel, G. E. Scuseria, M. A. Robb, J. R. Cheeseman, T. Vreven, K. N. Kudin, C. J. Burant, J. M. Millam, S. S. Iyengar, J. Tomasi, V. Barone, B. Mennucci, M. Cossi, G. Scalmani, N. Rega, G. A. Petersson, H. Nakatsuji, M. Hada, M. Ehara, K. Toyota, R. Fukuda, J. Hasegawa, M. Ishida, T. Nakajima, Y. Honda, O. Kitao, H. Nakai, M. Klene, X. Li, J. E. Knox, H. P. Hratchian, J. B. Cross, V. Bakken, C. Adamo, J. Jaramillo, R. Gomperts, R. E. Stratmann, O. Yazyev, A. J. Austin, R. Cammi, C. Pomelli, J. W. Ochterski, P. Y. Ayala, K. Morokuma, G. A. Voth, P. Salvador, J. Dannenberg, V. G. Zakrzewski, .Dapprich, A. D. Daniels, M. C. Strain, O. Farkas, D. K. Malick, A. D. Rabuck, K. Raghavachari, J. B. Foresman, J. V. Ortiz, Q. Cui, A. G. Baboul, S. Clifford, J. Cioslowski, B. B. Stefanov, G. Liu, A. Liashenko, P. Piskorz, I. Komaromi, R. L. Martin, D. J. Fox, T. Keith, M. A. Al-Laham, C. Y. Peng, A. Nanayakkara, M. Challacombe, M. Challacombe, P. M. W. Gill, B. Johnson, W. Chen, M. W. Wong, C. Gonzalez and J. A. Pople, Gaussian 03, Revision C.02, Inc., Wallingford CT (2004)

[25] W. L. Jorgensen, J. Chandrasekhar, J. D. Madura, R. W. Impey and M. L. Klein, Comparison of simple potential functions for simulating liquid water, J. Chem. Phys. 79, 926935 (1983).

[26] E. R. T. Tiekink, Crystal structure of 2-thoiuracil, Zeitschrift Kristall, 187, 79-84 (1989).

[27] S. W. Hawkinson, 1-Methyl-4-thiouracil, Acta Crystallogr B31, 2153-2155 (1975).

[28] P. Hobza, M. Kabelac, J. Sponer, P. Mejzlik and J. Vondrasek, Performance of empirical potentials (AMBER, CFF95, CVFF, CHARMM, OPLS, POLTEV), semiempirical quantum chemical methods (AM1, MNDO/M, PM3) and $a b$ initio Hartree-Fock method for interaction of DNA bases: Comparison with nonempirical beyond HartreeFock results, J. Comp. Chem. 18, 1136-1150 (1997).

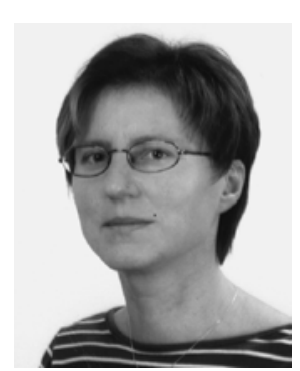

JoANNA SARZYŃSKa graduated from Warsaw University in biophysics. She obtained her Ph.D. at the Institute of Bioorganic Chemistry PAS, Poznan, studying an ionic exchange in solid state of nucleoside salts, by molecular spectroscopic methods. She then was a postdoctoral fellow in L. Nilsson laboratory at the Karolinska Institutet, Department of Biosciences at Novum, Center for Structural Biochemistry, Stockholm, Sweden, where she learned theoretical and simulation methods of study of biological molecules. Using molecular dynamics and free energy perturbation method, she contributed to the correlation of experimental biochemical and thermodynamic data for RNA fragments with structural flexibility and various interaction at the atomic level. She is currently a research associate in the Laboratory of Structural Chemistry of Nucleic Acids, Institute of Bioorganic Chemistry PAS, Poznan. Her research focuses on structure function relationships in unusual structural forms of RNA. In particular, she is interested in a study of an effect of base modification on the structure and stability of functionally important RNA elements. 